The SmartVision Navigation Prototype for Blind Users

J.M. Hans du Buf, João Barroso, João M.F. Rodrigues, Hugo Paredes, Miguel Farrajota, Hugo Fernandes, João José, Victor Teixeira, Mário Saleiro

International Journal of Digital Content Technology and its Applications Vol.5 No.5, May 2011

\title{
The SmartVision Navigation Prototype for Blind Users
}

\author{
${ }^{1}$ J.M. Hans du Buf, ${ }^{2,3}$ João Barroso, ${ }^{1}$ João M.F. Rodrigues, ${ }^{2,3}$ Hugo Paredes, \\ ${ }^{1}$ Miguel Farrajota, ${ }^{3}$ Hugo Fernandes, ${ }^{1}$ João José, ${ }^{3}$ Victor Teixeira, ${ }^{1}$ Mário Saleiro \\ 1, Corresponding Author Vision Laboratory - Inst. for Systems and Robotics (ISR), University of the \\ Algarve (FCT and ISE), Faro, Portugal, \{dubuf, jrodrig\}@ualg.pt \\ 2, GECAD, Knowledge Eng. and Decision Support Research Group, Inst. of Engineering of \\ Porto,Portugal, \{jbarroso, hugof\}@utad.pt \\ ${ }^{3,}$ University of Trás-os-Montes and Alto Douro (UTAD), Vila Real, Portugal \\ doi : 10.4156/jdcta.vol5.issue5.39
}

\begin{abstract}
The goal of the Portuguese project "SmartVision: active vision for the blind" is to develop a small, portable and cheap yet intelligent and reliable system for assisting the blind and visually impaired while navigating autonomously, both in- and outdoor. In this article we present an overview of the prototype, design issues, and its different modules which integrate GPS and Wi-Fi localisation with a GIS, passive RFID tags, and computer vision. The prototype addresses global navigation for going to some destiny, by following known landmarks stored in the GIS in combination with path optimisation, and local navigation with path and obstacle detection just beyond the reach of the white cane. The system does not replace the white cane but complements it, in order to alert the user to looming hazards. In addition, computer vision is used to identify objects on shelves, for example in a pantry or refrigerator. The user-friendly interface consists of a four-button hand-held box, a vibration actuator in the handle of the white cane, and speech synthesis. In the near future, passive RFID tags will be complemented by active tags for marking navigation landmarks, and speech recognition may complement or substitute the vibration actuator.
\end{abstract}

Keywords: Vision Aid, Autonomous Navigation

\section{Introduction}

The goal of the Portuguese SmartVision project is to develop and integrate technology into a portable device for aiding blind persons and those with severe visual impairments, about 180 million of which 40-50 million are completely blind. This device must be small, cheap and easy to assemble using off-the-shelf components. It must be extremely easy to carry and use, yet providing all necessary help for autonomous navigation. It should be stressed that the device cannot replace the white cane; it will be an extension of the cane, issuing warning signals when approaching a possible obstacle or when the footpath in front is curved and the heading direction should be adapted. The device cannot employ headphones as these block surround sounds. We assume that the user has adapted to the cane and relies on hearing. In this sense the device is "non-invasive" such that surround sounds and the device's signals, including verbal communications with queries and answers, are integrated in a natural way.

Sighted persons may have some difficulty in imagining the problems which blind persons must deal with almost all of the time, because they take the extremely comfortable efficiency of the visual system for granted. One brief glance suffices to determine the heading direction to a specific goal, to cross a street and to spot possible obstacles, both static ones like loose or missing cobblestones and steps in the pavement, also moving ones like persons and dogs. In a pantry, there is no problem distinguishing between cans of tomatoes and baked beans, even if both have the same size and both are red. Hence, the ultimate goal of the project is to substitute a significant part of the visual system, which is very ambitious.

Even the development of a device which mimics a guide-dog (Am.: seeing-eye dog) is a challenge. Such dogs have been trained to guide their owner around all obstacles and to recognise landmarks like certain shops, going from landmark to landmark, with subtle commands and hints resulting from extensive training. However, even a guide-dog is of little help when its owner needs to go to a location 
The SmartVision Navigation Prototype for Blind Users

J.M. Hans du Buf, João Barroso, João M.F. Rodrigues, Hugo Paredes, Miguel Farrajota, Hugo Fernandes, João José, Victor Teixeira, Mário Saleiro

International Journal of Digital Content Technology and its Applications Vol.5 No.5, May 2011

which the dog does not know: its function is reduced to local navigation, negotiating obstacles, footpaths and zebra crossings, that is, if the owner is already familiar with that location. If not, both are lost and the owner must ask passers-by, if there are any. An equivalent of a normal, printed map is required in order to not always have to rely on help by others and improve the autonomy of the user, with or without a dog.

From the above it is clear that we can distinguish three broad but linked applications: (1) local navigation for centering on footpaths etc. and obstacle avoidance, in the immediate surrounding but just beyond the reach of the white cane; (2) global navigation for finding one's way in the centre of the city, in a neighbourhood, or in a shopping centre; and (3) object recognition, not only cans etc. on shelves in a pantry or supermarket, but also outdoor: bus stops, taxi stands, cash machines (ATM) and telephone booths. Also banks, post offices and shops, simply all objects which have a distinct function and which can serve as a landmark for global navigation. Such objects could be recognised by their physical structure (image), by their specific sign, or by some sort of mark like an active RFID tag specifically employed for blind persons.

Below we discuss technological solutions in general (Section 2) and the ones being developed in the project: the prototype (Section 3), GIS and the navigation module (Section 4), the vision module (Section 5), the white cane with passive RFID tags (Section 6) and the interface module (Section 7). We conclude with a discussion in Section 8. It should be stressed that the functionality of the vision module for local navigation is detailed elsewhere [9].

\section{Technologies and Infrastructures}

Technology can provide solutions to the three broadly-defined applications, even with off-the-shelf hard- and software, but computing power may be a limiting factor in portable and therefore small devices. Another problem is the necessary infrastructure to use the technology, like outdoor GPS and indoor Wi-Fi reception in combination with a GIS, the level of detail of the latter, plus extra infrastructure specifically devoted to blind persons. For example, many railway stations have bands of rippled pavement at the platforms. Such bands can be employed at many other locations to mark the centres of footpaths. If too expensive, are there cheaper solutions? Since most roads are marked by white stripes which reflect light, it is also possible to mark footpaths, for example by reflecting circles a few metres apart. During the day these can be easily detected by a miniature camera, but during the night a small light source is necessary, for example a powerful LED, carried by the user, which flashes every two or three seconds.

A large metro and railway station in Lisbon is equipped with loudspeakers which emit sounds of birds, with different sounds in different areas for global navigation, and most sighted passengers do not even notice the sounds. Spoken messages stored in electronic tags are provided by the "Talking Points" system [14] and the tags can be fixed to anything, from an entry of a building to its elevators, also to bus stops and busses. This system is a commercial one, only providing "turnkey" solutions.

There are many possibilities, but the main question is: how much is a civilised society willing to invest in infrastructure for relatively few users, knowing that the infrastructure will make a tremendous difference for those users. For example, the reflecting circles mentioned above could be substituted by a solar-power driven LED in a small armature with a white-glass cover in the pavement, such that blind persons do not need to carry a light source when it is dark. One armature including a small solar panel, day-night sensor and other electronics might cost a few euro if mass produced. Electronic parts are so cheap that LEDs in special armatures can flash like a temporal barcode, too fast to be noticed by sighted persons, with codes linked to special places like footpath bifurcations and entries of buildings. However, it costs more to drill the holes and mount the armatures, unless the infrastructure is planned in new developments. Also, such armatures provide a solution when it is dark, but what during a sunny day when the temporal barcode cannot be detected?

An equivalent solution is to use RFID tags: passive tags without battery, which are cheap but have a small action radius, and active tags with battery or external (solar) power supply, which are more expensive but have a large action radius. To be useful, passive tags must be drilled into the pavement at rather small distances if the tip of the cane is equipped with a small sensor and the swaying cane should 
The SmartVision Navigation Prototype for Blind Users

J.M. Hans du Buf, João Barroso, João M.F. Rodrigues, Hugo Paredes, Miguel Farrajota, Hugo Fernandes, João José, Victor Teixeira, Mário Saleiro

International Journal of Digital Content Technology and its Applications Vol.5 No.5, May 2011

encounter the tags with a reasonable probability (see Section 6). Active tags, on the other hand, are more suitable for marking important landmarks like entries of buildings and shops, with a distance of 5 to 10 metres. Since both passive and active RFID tags emit unique codes, the codes can be stored in a database. Passive tags can be used for local navigation at footpath crossings etc., whereas active ones are better for global navigation, both in- and outdoor: the name of a landmark like a shop in the GIS for position calibration if GPS or Wi-Fi reception is not available. See also Section 3. In principle, GPS and Wi-Fi can also be supplemented by a realtime location tracking system if the user wears an active RFID tag [13], but this solution requires tag readers at many strategic locations.

Concerning object recognition in a supermarket or household pantry, if the blind person is wearing a camera at chest height, perhaps three shelves can be analysed with sufficient optical resolution. But in a supermarket these shelves normally carry the most expensive products, whereas the cheapest ones are on the bottom shelves. In addition, all shelves are cramped with products, because shelf space is money, hence products are not well separated and this hampers object segregation and identification (see Section 5). In a pantry, this problem can be avoided and both top and bottom shelves can be reserved for bigger things which can be easily identified by hand. In any case, it should be possible that a blind person takes an object in his hand, holds it at arm length in front of the camera attached at chest height, and uses a special command to ask the system to identify the object.

An alternative is to label objects with a QR barcode, a square one which can hold more information [1]. A mobile phone with a camera and QR barcode software sends the code to a URL server and receives an audio file, created by text-to-speech, with a description. Of course, this solution can be applied anywhere, software for printing and reading QR barcode labels is freely available, the price is almost nil apart from server and database maintenance, but the labels must always be visible. Instead of putting the labels on the products it is possible to put them on the shelves, provided they are checked on a regular basis and no one puts products at wrong locations. A complication when using a camera at chest height is the long distance between camera and barcode label, which also implies that there may be many labels in the camera's field of view. If a portable computer is used instead of a mobile phone, all software can be integrated and no wireless communications are required (text-to-speech may be required anyway; see Section 7).

For localising few but very important objects, like a bunch of keys at home or a suitcase on a conveyor belt at the luggage claim area of an airport, there already exist good solutions: an electronic label which can be attached to the object and a small hand-held gadget with a push button. Such technology works with ultrasound or radio signals, and the label beeps loudly when the button is pushed. It is rather easy to make a gadget with a few buttons for localising various objects. Here we may assume that blind persons do not put very important objects nor such a gadget at arbitrary locations, but: errare humanum est!

There are other technologies which can be employed, notably ultrasound detectors for spotting possible obstacles at foot- or head-height [10]. An alternative to GPS is Wi-Fi and mobile phone (GSM) localisation by "fingerprinting." Indoor, Wi-Fi is better than GSM, yielding an accuracy of 2.5-5.4 m, but outdoor GSM is better (75 $\mathrm{m}$ ), especially in urban areas with a high antenna density [16]. A recent development is Nokia's indoor positioning technology [4] based on DoA (direction-of-arrival), with an accuracy better than 2-5 m. Another technology employs ToA/AoA (time/angle of arrival) with UWB (ultra-wideband), aiming at $10 \mathrm{~cm}$. Pending availability of such advanced technology, one must select what is currently available and integrate this into a working system which is modular, such that future developments can be easily and rapidly adopted. With the current development in localisation technology, good solutions will be available soon and at many locations, both in- and outdoor. Below we describe the components of the SmartVision system. The approach for the Greek project SmartEyes [15] is similar to that of SmartVision, with ongoing collaboration, but SmartEyes' visual functionality is entirely based on stereo vision for detecting walkable open spaces and obstacles. For other initiatives we refer to $[5,10]$. 
The SmartVision Navigation Prototype for Blind Users

J.M. Hans du Buf, João Barroso, João M.F. Rodrigues, Hugo Paredes, Miguel Farrajota, Hugo Fernandes, João José, Victor Teixeira, Mário Saleiro

International Journal of Digital Content Technology and its Applications Vol.5 No.5, May 2011

\section{Prototype Overview}

The system integrates GPS (in the future the European Galileo equivalent) and Wi-Fi with a GIS. GPS is for outdoor navigation and Wi-Fi mainly for indoor, specifically in bigger buildings like a shopping centre or a university's administration. However, Wi-Fi access points are still not ubiquitous and GPS reception may not be available in narrow streets and in bad weather. Therefore, a fail-safe solution is required such that the user can still be assisted: by active RFID tags with calibrated positions, and if these cannot be located by visual object recognition, and, if all else fails, by the user asking a passer-by to bring him to the nearest landmark which is included in the GIS. The basic idea is that the user's system always has a global map with enough detail along the planned route. The actual position is constantly calibrated using GPS, Wi-Fi or RFID tags, whatever is locally available, and each time Wi-Fi reception is possible and a significant distance has been traversed, the local map is updated by the GIS server, for example in a radius of a few kilometres. This way the user can always consult the GIS for taking the best or shortest path to a destiny and for knowing the major landmarks along the route.

The system consists of a few modules; see Fig. 1. The central SmartVision module is responsible for managing all other modules and the communications with these. This module also receives input from the user (not shown) and takes decisions about what information the user should get from the system. Also not shown are the speech and other interfaces (see Section 7), nor is the electronic compass which will be integrated in the future.

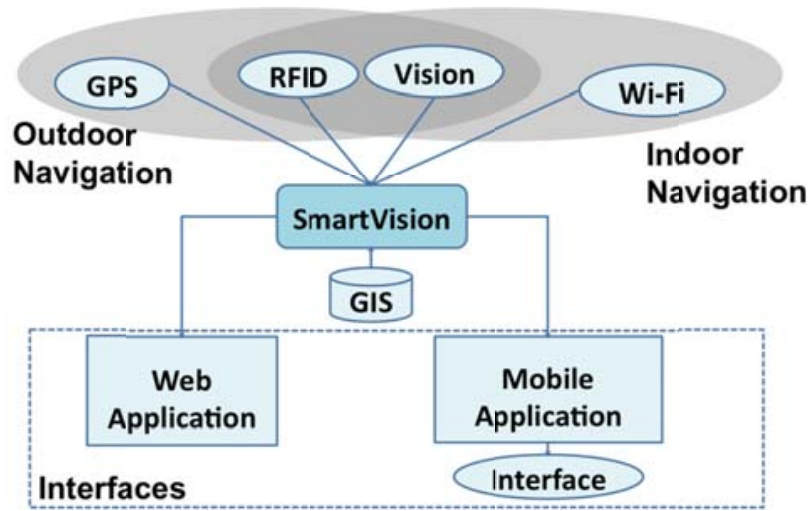

Figure 1. SmartVision prototype structure.

The GIS and navigation modules provide regular updates of the user's current geographic coordinates to the SmartVision module, either using GPS in outdoor or Wi-Fi in indoor environments. Both GPS and Wi-Fi are complemented by RFID tags and computer vision, and if GPS or Wi-Fi signals cannot be received the SmartVision module must rely on RFID tags and computer vision. The latter are common to both in- and outdoor environments and serve to detect specific landmarks. Each location technology has a specific accuracy and the navigation modules always choose the best accuracy from the ones which are available at any moment.

Concerning hardware, one small RFID reader can be placed in the tip of the white cane for detecting passive tags in the pavement, see Fig. 7, and the camera is chest-mounted. The GPS unit is connected by Bluetooth and Wi-Fi is a built-in component of the portable computer. In the future, a second RFID reader will be connected to the computer in order to detect active tags attached to landmarks.

The navigation modules are responsible for route planning and for providing information about surrounding points-of-interest (POIs). They connect to the SmartVision module and request GIS data and location data. To get the GIS data, the SmartVision module queries the GIS server in order to get a local map and its POIs. The user's position is obtained from the location modules. After analysing the requested data, the navigation modules feed the SmartVision module with navigation instructions. The 
The SmartVision Navigation Prototype for Blind Users

J.M. Hans du Buf, João Barroso, João M.F. Rodrigues, Hugo Paredes, Miguel Farrajota, Hugo Fernandes, João José, Victor Teixeira, Mário Saleiro

International Journal of Digital Content Technology and its Applications Vol.5 No.5, May 2011

amount and accuracy of the GIS data stored on the GIS server is critical in order to provide the best instructions.

The vision module provides local orientation instructions by detecting sidewalk borders and possible obstacles in front on the path, for guiding the user safely. It also detects already known landmarks (GIS in the immediate surrounding) in order to confirm encountered POIs and re-calibrate the user's position if GPS or Wi-Fi cannot be received. In addition, this module can be used to detect and recognise objects on demand. The camera used is the Bumblebee2 from Point Grey Research Inc. Being a stereo camera, it is possible to extract disparity information. This information is calibrated in order to estimate the distance between the user and a detected landmark (in the future to be combined with heading information from an electronic compass attached to the camera unit).

Finally, the interface module is the link between the SmartVision module and the user. At the moment it serves two outputs and one input. The two outputs are text-to-speech audio and vibration actuators. The vibration actuators are used for local navigation, i.e., obstacle avoidance and heading direction. The audio interface is used for navigating the menus and providing POI information. The user provides input by using a small four-button device to navigate a simple menu system and to select options. In a future version this can be replaced by speech recognition with pre-defined commands in combination with speech and sound synthesis.

\section{GIS and Navigation Modules}

Geographic Information Systems (GIS) are widely used as a powerful tool for storing, manipulating, visualising and analysing spatial data. GIS software enables users to view spatial data in a proper format. It also allows for the interpretation of spatial data in a way that is simple to understand by users with special needs, in this case blind persons and those with severe visual impairments.

Given the fact that Wi-Fi is not everywhere available, all or most information required for global navigation must be stored locally on the SmartVision prototype. This way it is possible to have access to the information whatever the scenario, both in- and outdoor, without a need to regularly query the GIS server. The geographic information stored on the prototype is updated when Internet connection is available, through the use of Web services. The information is stored in digital map files, or shapefiles [8], and a MySQL database which holds landmark details. For the distribution of geographic information, the adopted architecture is three-tier client/server [12]. This way, functionalities can easily be shared by multiple applications, the processing can be distributed over powerful servers to achieve performance requirements, and the separation of logical components facilitates management of the database. In our system, the client application, i.e., the SmartVision prototype, handles and provides geographic information to the user. For a detailed description see [2, 8]. Figure 2 illustrates the client/server architecture.

The user triggers the updating of the local map using the interface module. When triggered, the client application sends a request to the Web server, which forwards it to the GIS server. The GIS server then interprets the request and retrieves the spatial data stored in the shapefiles to generate a map. This map is sent back to the Web server, which, in turn, sends it to the client application (i.e., the SmartVision prototype). Examples of mapped locations are listed in Table 1.

In order to manage the data stored in the GIS, the most basic management tasks involved are periodic updates of the database and/or shapefiles by a system administrator. To simplify the task of viewing and editing the raw data present on the GIS server, as well as to reduce the need of specialised staff, a web application is used for content management. This application includes both a front office (web portal) and back office (for content management by a system administrator). This application is available by accessing the Web server. Therefore, the Web server fulfills two roles: content management and data transfer to the SmartVision prototype.

In the case of outdoor locations, our experience indicates that geocoding is best done manually using an aerial view. However this can pose some problems in getting coordinates of some specific points due to photo resolution. GPS is being used to fix those points. 
The SmartVision Navigation Prototype for Blind Users

J.M. Hans du Buf, João Barroso, João M.F. Rodrigues, Hugo Paredes, Miguel Farrajota, Hugo Fernandes, João José, Victor Teixeira, Mário Saleiro

International Journal of Digital Content Technology and its Applications Vol.5 No.5, May 2011

The navigation modules handle aspects related to the computation of the route that the user must follow, from an initial or actual position to the chosen destiny. In terms of functionality, all local POIs in the database are transmitted to the user by the interface module, in order to choose a desired one.

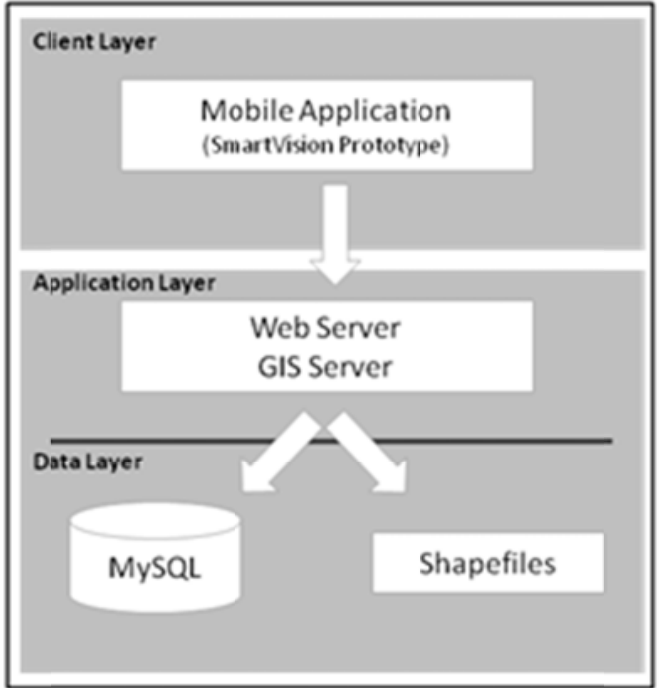

Figure 2. Three-tier client/server architecture.

Table 1. Examples of the locations stored in the GIS server.

\begin{tabular}{cc}
\hline Outdoor & Indoor \\
\hline Access roads & Offices \\
Roads & Classrooms \\
Road signs & Lavatories \\
Number of parking places & Services \\
Car parkings & Wi-Fi APs \\
Buildings & \\
Crosswalks & \\
Green zones & \\
Sport facilities & \\
Bus stops & \\
\hline
\end{tabular}

Navigation instructions are issued using Dijkstra's Shortest Path First (SPF) routing algorithm; see Fig. 3. According to Ertl [6], this algorithm is able to calculate, in a graph, the shortest path from a starting vertex to a destination vertex, and this algorithm provides a balanced solution between calculus efficiency and implementation simplicity. Obviously, the road layer in the map is built in a manner similar to a graph, i.e., the points where a road begins, is intersected and ends correspond to the graph's vertices and these are linked by the graph's edges. See [2, 8] for further details. The current version of the GIS of UTAD campus counts 53 roads, 32 zebra crossings, 34 buildings with entries, $6 \mathrm{Wi}-\mathrm{Fi}$ access points and 5 indoor spaces, and more are still being added.

Concerning the use of Wi-Fi, this technology is also used to compensate for the lack of GPS reception inside buildings. By using the calibrated locations of Wi-Fi access points (APs) in the GIS in combination with a triangulation method based on the APs' signal strengths, it is possible to approximate the user's position with an error margin of 7-8 m, which is worse than Nokia's margin of 2-5 m [4]. Implicitly we assume that there are at least three APs within reception range, i.e., at the time the localisation by Wi-Fi is requested. This solution provides an indoor localisation system very similar to outdoor GPS. However, if less than three APs are within reception range, an alternative to recalibrate the user's actual position is required. Therefore, in the near future tests with active RFID tags 
The SmartVision Navigation Prototype for Blind Users

J.M. Hans du Buf, João Barroso, João M.F. Rodrigues, Hugo Paredes, Miguel Farrajota, Hugo Fernandes, João José, Victor Teixeira, Mário Saleiro

International Journal of Digital Content Technology and its Applications Vol.5 No.5, May 2011

will be conducted in combination with visual landmark (object) recognition by the Vision module. These extensions are required in any case, i.e., if no or few APs are present inside a building or GPS is temporarily not available outdoor.

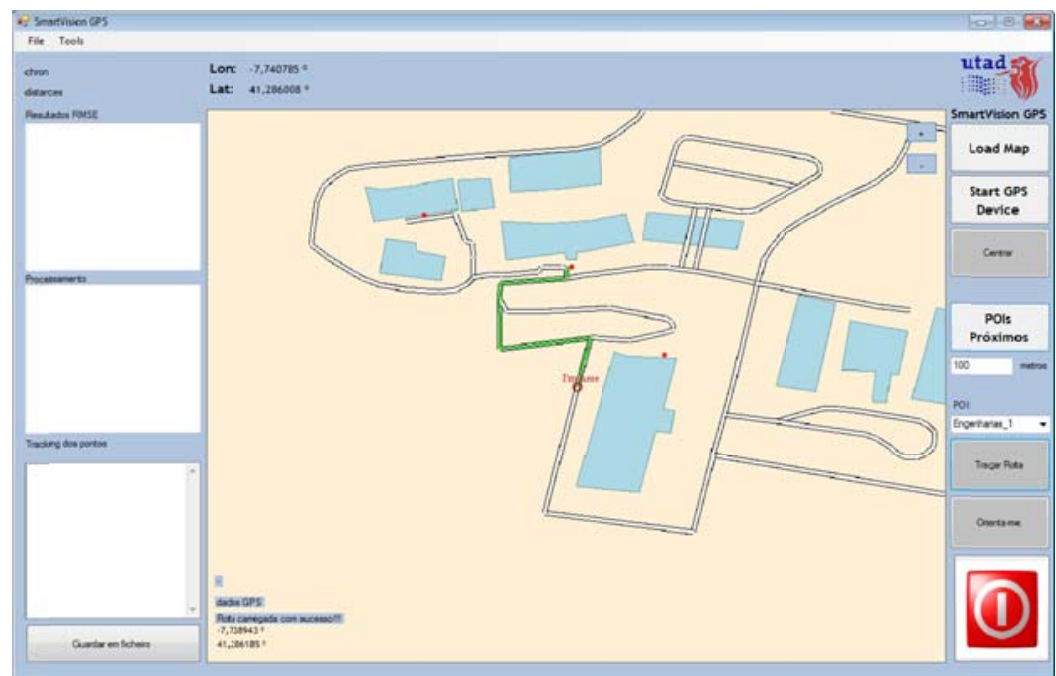

Figure 3. Example of the application of Dijkstra’s SPF algorithm to path finding in part of the GIS of UTAD campus.

\section{Vision Module}

The Vision module provides information for local navigation: the heading direction to keep the user within safe boundaries in corridors and on paths including footpaths (sidewalks), possible obstacles in front and just beyond the reach of the white cane, and known objects which may serve as navigation landmarks or which can be a destiny (bus stop, taxi stand, cash machine, post office, etc.). The latter aspect must still be studied by using real test video sequences, although first results on the recognition of household items are available. This module consists of four major sub-modules: sidewalk and path detection, obstacle detection, detection of moving persons or other objects possibly on collision course, and object recognition; see Fig. 4.

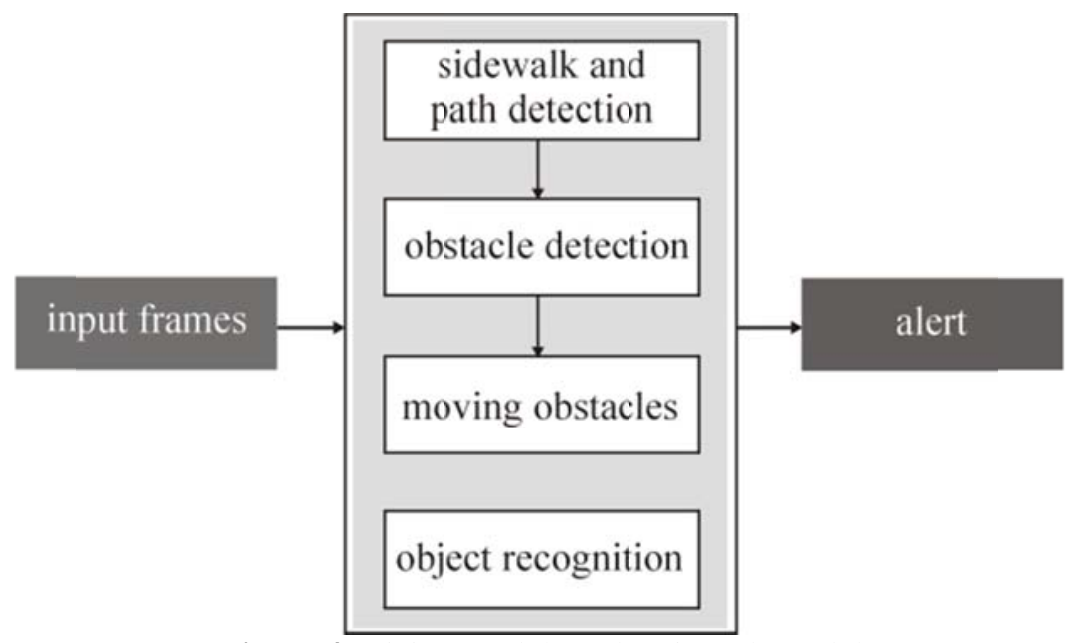

Figure 4. Vision module and major sub-modules. 
The SmartVision Navigation Prototype for Blind Users

J.M. Hans du Buf, João Barroso, João M.F. Rodrigues, Hugo Paredes, Miguel Farrajota, Hugo Fernandes, João José, Victor Teixeira, Mário Saleiro

International Journal of Digital Content Technology and its Applications Vol.5 No.5, May 2011

Algorithms for path detection and obstacle avoidance are detailed in [9]. These are similar to those used for robot navigation in corridors [17]. Figure 5 shows examples of two sequences in which a looming obstacle has been detected and an obstacle alert has been generated to inform the user.
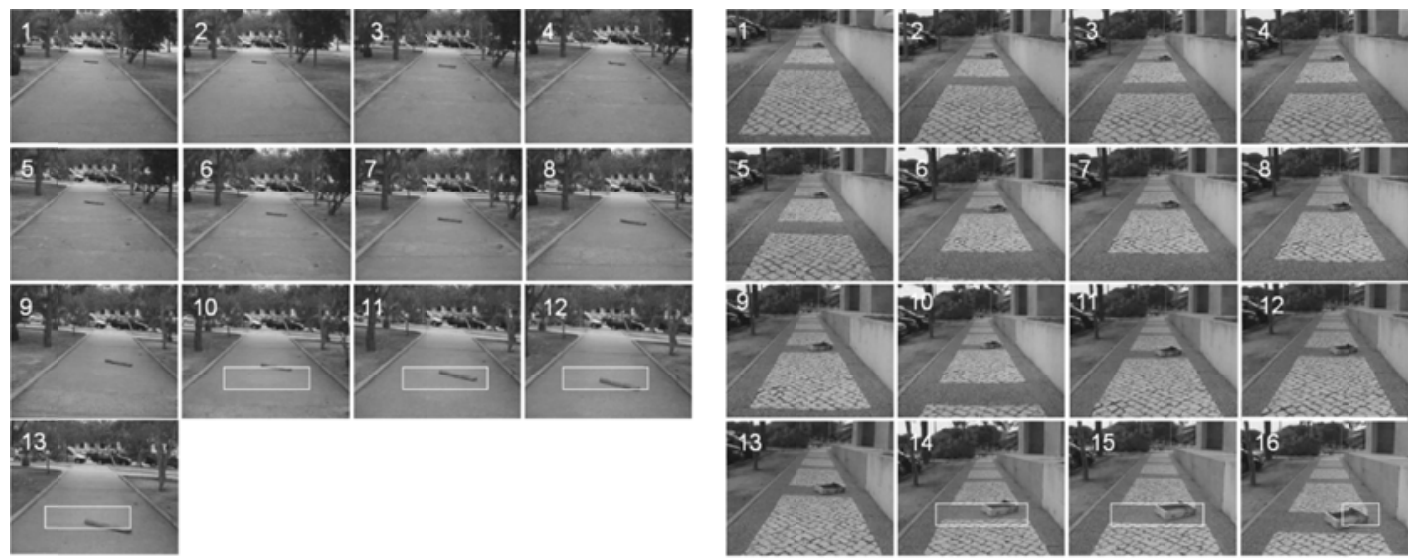

Figure 5. Two sequences with different pavements and detected obstacles.

An important goal is to locate household items, for example on shelves in a pantry, like toothpaste or a bottle of ketchup. To this purpose we use the well-known algorithm coined SURF (Speeded Up Robust Features), which is a scale- and rotation-invariant interest-point detector and descriptor [3]. Bay et al. [3] claim that it outperforms previously proposed schemes like SIFT with respect to repeatability, distinctiveness and robustness, and that it is faster. All this is achieved by relying on integral images for image convolutions and on the strengths of the best existing detectors and descriptors - Hessian matrix-based detector and distribution-based descriptor - simplifying these methods to the essential.

Extensive tests are being conducted with many typical household objects, using the OpenSURF library, a $\mathrm{C} / \mathrm{C}++$ implementation. Depending on the complexity of each object, a single or a few different views are used for training, their features being used in realtime for locating and identifying the objects arranged on shelves [5]. As expected, the recognition rate is very good, provided that the camera is at reasonably short distance, about $0.5 \mathrm{~m}$. Of course, the user must be trained in holding and pointing the camera, also in communicating with the system in order to optimise performance. Figure 6 shows examples of shelves in a pantry and a refrigerator with identified objects. In the future, similar tests will be conducted to recognise bigger but important objects for navigation, first indoor (elevator, lavatory, welcome desk, furniture, plants) and then outdoor (cash machine, telephone booth, etc.).
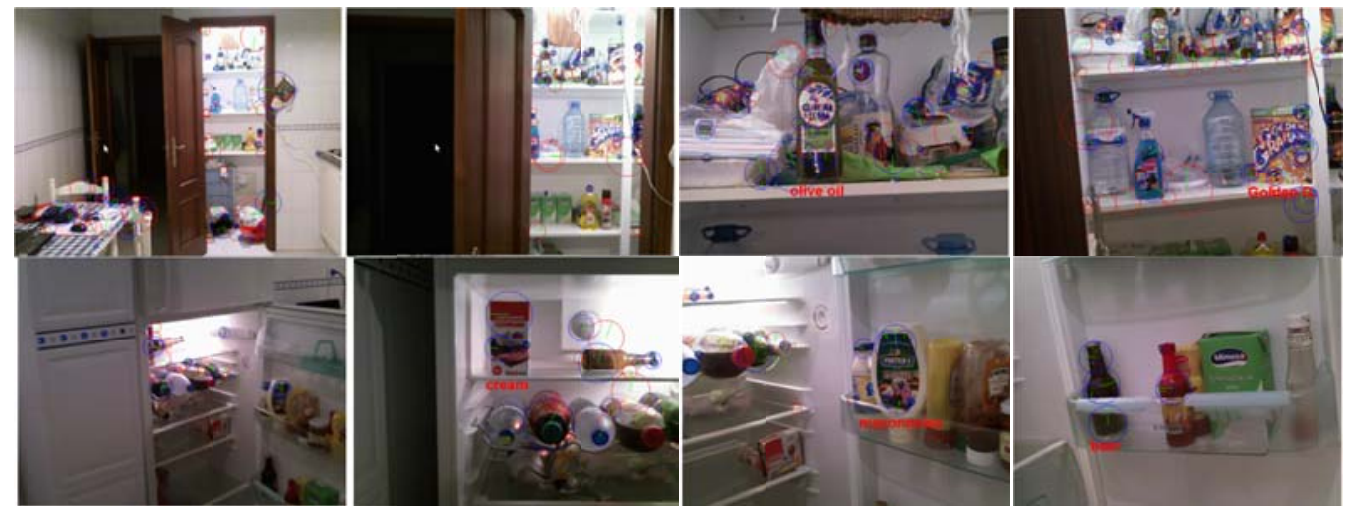

Figure 6. Typical views of household items on shelves in a pantry and a refrigerator which have been located and recognised using the OpenSURF software. 
The SmartVision Navigation Prototype for Blind Users

J.M. Hans du Buf, João Barroso, João M.F. Rodrigues, Hugo Paredes, Miguel Farrajota, Hugo Fernandes, João José, Victor Teixeira, Mário Saleiro

International Journal of Digital Content Technology and its Applications Vol.5 No.5, May 2011

\section{White Cane and Passive RFID Tags}

Above, the use of white markers on the pavement for aiding local navigation was discussed. One of the main problems of such markers is the necessity of a light source when it is dark. An alternative solution is to use passive RFID tags in the pavement, which always respond provided that a signalemitting antenna is at close range, less than $10 \mathrm{~cm}$. Experiments were conducted with an RFID antenna in the very tip of a cane made of light PVC, similar to the ones used by blind persons. The handle contains a vibration actuator and close to the handle was mounted the tag reader with Bluetooth interface; see Fig. 7 (top-left) and [7].

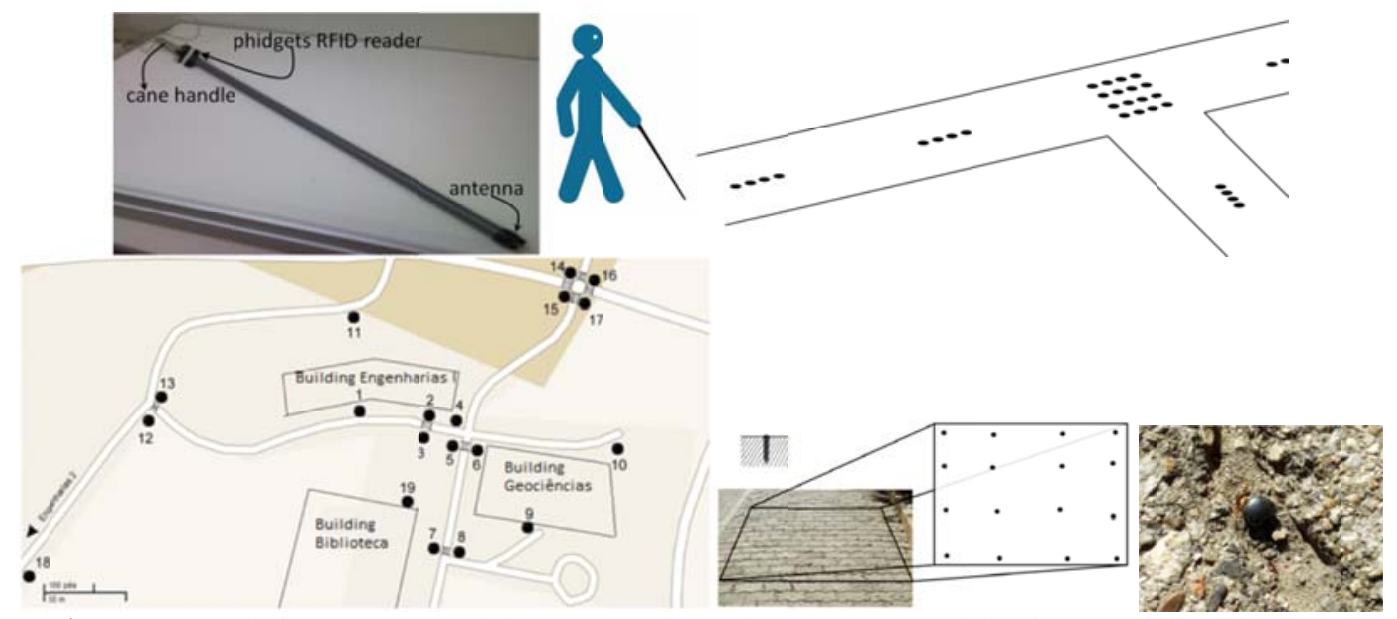

Figure 7. Top-left: PVC cane with RFID reader and antenna. Top-right: layout of the tags on paths. Bottom, from left: tag clusters at a part of UTAD campus, a tag cluster, and a tag planted in the ground.

The RFID tag reader used is from Phidgets, mainly because of previous experience and easy implementation. The passive tags have a working frequency of $135 \mathrm{kHz}$. The tags have the shape of a nail or golf tee, $2.5 \mathrm{~cm}$ long and $4 \mathrm{~mm}$ wide, and the electronics are fixed inside a hard and resistant plastic encapsulation [5]. The tags can be easily inserted into drilled holes in the pavement.

In experiments we tested different tag layouts, both in a laboratory and on UTAD Campus, in order to find the best layout. The main problem is the combination of the walking speed of the user, the swaying of the cane and the distance of the cane's tip to the surface of the pavement, i.e., the probability of "hitting" tags with some regularity. Initially the tags were distributed on the pathway along three parallel lines: two lines close to the path borders and one line in the centre to alert the user to special events like fixed obstacles (trash cans, trees) and change of direction. To this end the codes of the tags are stored in a small database together with event types, and the cane's vibrator is activated with different temporal patterns. In a second configuration, a single line along the path's centre was tested in combination with four-by-four tag arrays at bifurcations etc.; see Fig. 7 (top-right). The latter solution proved to be better, only requiring additional tests in order to optimise the tags' spacing for increasing the "hit rate." Results of the tests and further details are described in [7]. Figure 7 (bottom) shows positions of rectangular tag clusters at UTAD campus and a photograph of a tag.

\section{Interface Module}

When developing a navigation aid for visually impaired users, the user interface has to be intuitive and extremely easy to use [11]. The interface can only rely on touch and hearing, but it may not interfere with normal hearing of surround sounds nor the normal use of the white cane. The actual version of the SmartVision prototype employs speech synthesis, a vibration actuator in the handle of the cane, and a small hand-held box with only 4 pushbuttons. Each interface has a specific use. The 
The SmartVision Navigation Prototype for Blind Users

J.M. Hans du Buf, João Barroso, João M.F. Rodrigues, Hugo Paredes, Miguel Farrajota, Hugo Fernandes, João José, Victor Teixeira, Mário Saleiro

International Journal of Digital Content Technology and its Applications Vol.5 No.5, May 2011

speech module is used to guide the user with brief messages, or longer messages but only when intentionally requested by the user. The vibration module is used when very simple instructions like "turn left" or "turn right" are issued.

At the moment the pushbutton box is the only input device to the prototype. Linked to the computer by a simple USB interface, different combinations of the four buttons allow the user to request information and to navigate through a simple menu system [8]. For instance, the user is able to ask for detailed information about the surrounding environment, or only one specific landmark. If available, this information will be provided by the speech module. The speech module consists of a Dynamic Link Library (DLL) which uses the Microsoft speech synthesizer API. This DLL also allows to select the voice and output device, and to control volume and pitch. In spite of providing all necessary or available information for guidance, the audio interface is limited to speech synthesis. In principle, speech recognition can also be employed, but may be less reliable near roads with heavy traffic or at sites with loud music or many talking people.

\section{Discussion}

In this article we described the current version of the SmartVision prototype, which integrates a GIS with positioning by GPS and Wi-Fi, also computer vision for path, obstacle and object detection. In the near future, active RFID tags will be integrated for landmark detection if GPS and Wi-Fi reception are not possible, as will be an electronic compass in order to know the actual heading direction of the user and to give instructions to correct the direction. The latter is for global navigation because the vision module handles local navigation for negotiating paths and obstacles.

The GIS of UTAD campus [8] has been prepared, but it will be refined and extended by a few indoor locations to test also navigation in big buildings. The visual functions will be extended by stereo vision in order to estimate distances to objects and obstacles, and visual recognition of important landmarks, which extends recognition of household items on shelves etc., is already being explored.

The FCT-funded project SmartVision will terminate mid-2011, but a follow-up project of two years has already been approved. This extension suffices to optimise all modules and to include the newest and best localisation solutions like Nokia's indoor one with a better precision. In addition, extensive field tests are planned in collaboration with ACAPO, the Portuguese association for amblyopes and the blind. The goal is to convert the prototype into a real system which can be assembled and maintained by non-experts, with detailed instructions. This system is coined Blavigator, from blind navigator.

\section{Acknowledgements}

This research was supported by the Portuguese Foundation for Science and Technology (FCT), through the pluriannual funding of the Institute for Systems and Robotics (ISR/IST) through the PIDDAC Programme funds, and by the FCT project SmartVision: active vision for the blind (PTDC/EIA/73633/2006).

\section{References}

[1] Hend S. Al-Khalifa "Utilizing QR code and mobile phones for blinds and visually impaired people" In Proc. 11th Int. Conf. on Computers Helping People with Special Needs, Springer, LNCS 5105, pp. 1065-1069, 2008.

[2] José Almeida, Hugo Fernandes, Vitor Filipe, João Barroso "Web platform architecture to support the geographic information system of the University of Trás-os-Montes and Alto Douro Campus" In Proc. Int. Conf. on New Trends in Information and Service Science, pp. 1112-1117, 2009.

[3] Herbert Bay, Andreas Ess, Tinne Tuytelaars, Luc Van Gool "Surf: Speeded up robust features" Computer Vision and Image Understanding, Vol. 110, No. 3, pp. 346-359, 2008.

[4] Fabio Belloni, Ville Ranki, Antti Kainulainen, Andreas Richter “Angle-based indoor positioning system for open indoor environments” In Proc. 6th Worksh. on Positioning, Navigation and Communications, pp. 261-265, 2009. 
The SmartVision Navigation Prototype for Blind Users

J.M. Hans du Buf, João Barroso, João M.F. Rodrigues, Hugo Paredes, Miguel Farrajota, Hugo Fernandes, João José, Victor Teixeira, Mário Saleiro

International Journal of Digital Content Technology and its Applications Vol.5 No.5, May 2011

[5] J.M. Hans du Buf, João Barroso, João M.F. Rodrigues, Hugo Paredes, Miguel Farrajota, Hugo Fernandes, João José, Victor Teixiera, Mário Saleiro “The SmartVision navigation prototype for the blind” In Proc. Int. Conf. on Software Development for Enhancing Accessibility and Fighting Info-exclusion (DSAI), pp. 167-174, 2010.

[6] Gerhard Ertl "Shortest path calculation in large road networks” OR Spektrum, Vol. 20, No. 1, pp. 15-20, 1998.

[7] José Faria, Sérgio Lopes, Hugo Fernandes, Paulo Martins, João Barroso “Electronic white cane for blind people navigation assistance” In Proc. World Automation Congress, pp. 1-7, 2010.

[8] Hugo Fernandes, Telmo Adão, Nuno Conceição, Hugo Paredes, Pedro Araújo, João Barroso "Using GIS platforms to support accessibility: the case of GIS UTAD” In Proc. Int. Conf. on Universal Technologies, pp. 71-80, 2010.

[9] João José, Miguel Farrajota, João M.F. Rodrigues, J.M. Hans du Buf “The SmartVision local navigation aid for blind and visually impaired persons” JDCTA: Int. J. of Digital Content Technology and its Application, 2011 (Accepted).

[10] Laehyun Kim, Sehyung Park, Sooyong Lee, Sungdo Ha "An electronic traveler aid for the blind using multiple range sensors” IEICE, Electronics Express, Vol. 11, No. 6, pp. 794-799, 2009.

[11] Ryszard Kowalik, Stanislaw Kwasniewski "Navigator - A talking GPS receiver for the blind" In Proc. Computers Helping People with Special Needs, Springer, LNCS 3118, pp. 446-449, 2004.

[12] Zhong-Ren Peng, Ming-Hsiang Tsou "Internet GIS: distributed geographic information services for the internet and wireless networks” John Wiley \& Sons Inc, New Jersey, 2006.

[13] Joseph K. Siror, Liang Guanqun, Kaifang Pang, Sheng Huanye, Wang Dong "Use of RFID for intelligent pre-shipment inspection" JDCTA: Int. J. of Digital Content Technology and its Applications, Vol. 4, No. 8, pp. 242-251, 2010.

[14] Jason Stewart, Sara Bauman, Michelle Escobar, Jakob Hilden, Kumud Bihani, Mark Newman “Accessible contextual information for urban orientation” In Proc. 10th Int. Conf. on Ubiquitous Computing, pp. 332-335, 2008.

[15] Nikolaos Trichakis, Dimitrio Bisias, Styliani Taplidou, Eleni Korkontzila, Leontios Hadjileontiadis "SmartEyes: an enhanced orientation and navigation system for blind or visually impaired people” IEEE Computer Society, CSIDC 2003 Final Report, pp. 1-20, 2003.

[16] Alex Varshavsky, Mike Chen, Eyal de Lara, Jon Froehlich, Dirk Haehnel, Jeffrey Hightower, Anthony LaMarca, Fred Potter, Timothy Sohn, Karen Tang, Ian Smith “Are GSM phones the solution for localization?” In Proc. 7th IEEE Workshop on Mobile Computing Systems and Applications, pp. 20-28, 2006.

[17] Zhengyin Zhou, Tianding Chen, Di Wu, Changhong Yu "Corridor navigation and obstacle distance estimation for monocular vision mobile robots" JDCTA: Int. J. of Digital Content Technology and its Applications, Vol. 5, No. 3, pp. 192- 202, 2011. 